\title{
Mechanische Obstruktion, paralytischer Ileus, Ileuskrankheit und postoperativer Ileus
}

\author{
M. H. Müller, K. S. Lehmann, M. E. Kreis \\ Klinik für Allgemein-, Thorax- und Gefäßchirurgie; Charité Berlin - Campus Benjamin Franklin
}

Der lleus stellt eine der häufigsten Notfalldiagnosen im chirurgischen Alltag dar. Es wird angenommen, ohne das hierfür belastbare Zahlen vorliegen, dass bei ca. $10 \%$ der Notfallaufnahmen wegen einer Abdominalsymptomatik in die deutschen Rettungsstellen der Krankenhäuser ein lleus vorliegt. Es handelt sich somit um ein relevantes Problem im klinischen Alltag. Wird der lleus nicht rechtzeitig erkannt und therapiert, kann sich daraus ein potenziell lebensbedrohliches Krankheitsbild entwickeln. Jeder klinisch tätige Kollege sollte deshalb mit der Diagnostik, Therapie und dem Verlauf der Erkrankung vertraut sein. In den folgenden Abschnitten soll die Erkrankung in all ihren Facetten unter Berücksichtigung der aktuellen Literatur systematisch aufgearbeitet werden.

\section{Definition}

Die Definition des Ileus ist leider sehr unscharf und subsumiert eine Reihe von Störungen. Im angloamerikanischen Sprachgebrauch wird der Begriff Ileus lediglich für Paralyse des Darms gebraucht. Der mechanische Ileus wird hier als Obstruktion bezeichnet. Im folgenden Artikel soll vor allem auf die mechanische Obstruktion eingegangen werden. Allerdings ist der paralytische lleus häufig eine Komponente der mechanischen Obstruktion und der sich im Verlauf entwickelnden Ileuskrankheit. Um beide Begriffe scharf voneinander trennen zu können, werden im gesamten Text die Begriffe mechanische Obstruktion oder paralytischer Ileus konsistent verwendet.

Der postoperative Ileus, als weitere Sonderform der Erkrankung, ist im weitesten Sinn ein paralytischer Ileus,

\section{Abkürzungen}

$\begin{array}{ll}\text { CT } & \text { Computertomografie } \\ \text { NO } & \text { Stickstoffmonoxid } \\ \text { NANC } & \text { nonadrenerg, noncholinerg } \\ \text { POI } & \text { postoperativer lleus }\end{array}$

welcher durch das operative Trauma von z. B. abdominalchirurgischen Eingriffen verursacht wird. Die Pathophysiologie dieser Sonderform des Ileus ist experimentell in den letzten Jahren gut untersucht worden und wird im letzten Abschnitt ausführlich beschrieben.

\section{Ätiologie}

Die mechanische Obstruktion wird in der Regel durch ein mechanisches Hindernis ausgelöst, welches schließlich zur Okklusion des Darmlumens führt. Als Ursache kommen z. B. Briden, Adhäsionen, Tumoren, Fremdkörper sowie Gallensteine in Betracht. Eine mechanische Obstruktion kann aber auch durch eine Einklemmung des Darms im Rahmen einer inkarzerierten Hernie, eines Volvulus oder einer Invagination entstehen. Die Patienten, die diese Form der mechanischen Obstruktion entwickeln, zeigen häufig das Bild eines akuten Abdomens.

Eine weitere Möglichkeit der Differenzierung kann anhand der Lokalisation erfolgen. So können wir zwischen Obstruktionen, die den Dünndarm oder den Dickdarm betreffen, unterscheiden. Inkarzerierte Hernien oder Briden nach vorangegangenen chirurgischen Eingriffen führen häufig zu einer Obstruktion im Bereich des Dünndarms. Als weitere wichtige Ursache für eine mechanische Dünndarmobstruktion ist die Peritonealkarzi- 


\section{Hintergrund}

\section{Verwachsungen und Briden}

Es ist anzunehmen, dass die Zahl an Verwachsungen und Briden aufgrund des Einsatzes laparoskopischer Operationstechniken in Zukunft abnehmen wird. Dieser Effekt wird höchstwahrscheinlich durch eine Zunahme an Obstruktionen durch Tumorerkrankungen in einer alternden Gesellschaft wieder ausgeglichen werden.

nose zu nennen. Diese ist häufig mit Tumoren des kolorektalen Karzinoms, Ovarialkarzinoms oder auch des Magenkarzinoms vergesellschaftet. Da der Dünndarm dem Dickdarm vorgeschaltet ist, kann auch eine Dickdarmobstruktion konsekutiv zu einem Dünndarmproblem führen.

\section{Pathophysiologie}

Der paralytische lleus zeichnet sich durch eine verminderte Funktion der Darmwandmuskulatur aus, was in einer verringerten oder aufgehobenen Motilität resultiert. Die primäre Form des paralytischen Ileus ist selten und kommt bei Myopathien oder Neuropathien vor. Die

Tabelle 1

Ursachen des paralytischen lleus.

Art der Ursache Ursachen

reflektorisch postoperativ nach abdominellen oder retroperitonealen Operationen, retroperitoneales Hämatom, Frakturen (z. B. Wirbelkörper), Harnverhalt, Gallenkolik, Nierensteine, Stieldrehung des Ovars, Hodentorsion, Ogilvie-Syndrom

infektiös intra- oder retroperitoneale Infektionen (z. B. bakterielle Peritonitis, intraabdominelle Abszesse), pseudomembranöse Kolitis, entzündliche Darmerkrankungen, bakterielle oder parasitäre Darminfektionen, Pankreatitis

medikamentös Opioide, Katecholamine, Antidepressiva, Neuroleptika mit anticholinerger Wirkung

metabolisch Urämie, Elektrolytstörungen (insbesondere Hypokaliämie), diabetische Azidose

toxisch Endstadium des mechanischen lleus, Megakolon bei Colitis ulcerosa, Pankreatitis, Bleivergiftung

vaskulär arterielle mesenteriale Ischämie (Mesenterialinfarkt), nicht okklusive mesenteriale Ischämie wesentlich häufigere sekundäre Form kann neben der mechanischen Obstruktion eine Vielzahl von Ursachen haben (Tab. 1).

Krankheitsverlauf bis zum Vollbild der Ileuskrankheit. Dem paralytischen Ileus liegt eine herabgesetzte oder ungeordnete Aktivität von Myozyten der Darmwand zugrunde. Die resultierende Dysmotilität führt zu einer Stase von Flüssigkeit und Darminhalt. In Folge entsteht ein erhöhter intraluminaler Druck mit intestinaler Dilatation. Es kommt zu einer inflammatorischen Reaktion mit Invasion neutrophiler Granulozyten in die Muskulatur der Darmwand und zu einer Freisetzung von Stickstoffmonoxid (NO). Durch die dadurch hervorgerufene Schädigung der Myozyten wird die intestinale Dilatation im Sinne eines Circulus vitiosus weiter verschärft. Der Anstieg der Darmwandspannung führt zur Schädigung der Mikrozirkulation und zu einem Darmwandödem. Flüssigkeit strömt in das Darmlumen und führt zur Hypovolämie und Elektrolytstörungen. Die intestinale Stase führt zudem zu einer vermehrten bakteriellen Überwucherung. Aufgrund des hypoxiebedingten $\mathrm{Zu}-$ sammenbruchs der Mukosabarriere findet eine vermehrte bakterielle Dislokation, systemische Endotoxineinschwemmung und Durchwanderungsperitonitis statt. Es kann sich schließlich das Vollbild der Ileuskrankheit mit Hypovolämie, septischem Schock und Multiorganversagen entwickeln. Die Flüssigkeits- und Elektrolytverluste werden durch das hypomotilitätsbedingte Erbrechen weiter verstärkt.

Die Folgeprobleme der lleuskrankheit können verschiedene Organsysteme betreffen:

- hämodynamische Instabilität durch Hypovolämie

- abdominelles Kompartmentsyndrom

\section{Hintergrund}

\section{Postoperativer lleus}

Eine Sonderstellung nimmt der postoperative lleus ein. Durch die Manipulation bei einer Laparotomie wird eine Entzündungskaskade aktiviert, die eine transiente Verminderung der muskulären Kontraktilität bewirkt. Diese Form des paralytischen lleus tritt regelmäßig nach größeren abdominellen, retroperitonealen, neurochirurgischen und orthopädischen Operationen auf und sollte innerhalb von 72 Stunden selbstlimitierend sein. Die Pathogenese des postoperativen lleus wurde in den letzten Jahren umfassend grundlagenwissenschaftlich untersucht und würde den Umfang dieser Abhandlung sprengen. 
- erhöhter intrathorakaler Druck infolge der abdominellen Distension mit verschlechterter Atemmechanik

- Minderperfusion der Leber durch venöse und portalvenöse Druckerhöhung

- renale Minderperfusion mit Niereninsuffizienz

- Aspirationspneumonie bei Erbrechen

\section{Klinische Symptomatik}

Typische Zeichen der mechanischen Obstruktion sind kolikartige abdominelle Schmerzen, Übelkeit und Erbrechen sowie Stuhlverhalt. Es gilt jedoch zu beachten, dass, je nach Lokalisation der mechanischen Obstruktion, Übelkeit und Erbrechen oder aber auch der Stuhlverhalt fehlen können. So kann bei einem hohen Dünndarmileus die Darmpassage noch intakt sein, dagegen bei einer mechanischen Obstruktion im Bereich des distalen Kolons Übelkeit und Erbrechen fehlen.

Beim paralytischen lleus findet man gehäuft Singultus, schwallartiges Erbrechen und Stuhlverhalt. Das Abdomen ist meist distendiert. Auskultatorisch fehlt die Peristaltik (sog. Totenstille). Im Gegensatz zum mechanischen Ileus liegen keine kolikartigen Schmerzen vor, sondern es überwiegt häufig ein diffuser Bauchschmerz. Die Bauchdecke kann weich sein, oder aber bei bereits stattgefundener Durchwanderung oder einer auslösenden Peritonitis bretthart sein.

Die zugrunde liegenden Ursachen der mechanischen Obstruktion und des paralytischen lleus können unterschiedlich sein. Sie haben jedoch gemeinsam, dass sie, sollte nicht rechtzeitig interveniert werden, in die gemeinsame Endstrecke der Ileuskrankheit münden. Dabei kommt es unter Vermittlung des intrinsischen und extrinsischen Nervensystems des Gastrointestinaltrakts zu Elektrolytstörungen, die nachfolgend zu Hypovolämie mit Tachykardie und Hypotonie führen können.

- Im weiteren Verlauf sind die Symptome der Folgeprobleme zu beobachten, wie Anurie, Kreislaufinstabilität oder Dyspnoe. Bei Persistenz der Passagestörung kommt es zu Translokation von Darmkeimen durch die Darmwand oder noch gravierender zur Perforation des Darms, was zu einer massiven Entzündungsreaktion, generalisierter Peritonitis bis hin zum Vollbild des septischen Schocks führen kann.

\section{Diagnostisches Vorgehen}

Aufgrund der Schwere des Krankheitsbilds benötigen Patienten, die sich mit dem Bild einer mechanischen Obstruktion oder des paralytischen Ileus präsentieren, eine dringliche Abklärung. Die Diagnostik beider Krankheitsbilder ist im Wesentlichen identisch. Hierzu zählen Blutuntersuchungen, interventionelle Untersuchungen sowie bildgebende Verfahren.

Spezifische Laborparameter existieren nicht. Die typische Labordiagnostik umfasst ein Blutbild (Hämatokrit, Leukozytose), Elektrolyte (insbesondere Hypokaliämie), Kreatinin und Harnstoff (Niereninsuffizienz, Urämie), Gerinnung und eine Blutgasanalyse (Säure-Basen-Status, Laktat).

Die Abdomenübersicht im konventionellen Röntgen hat eine hohe Sensitivität von bis zu 98\% in der Diagnosestellung. Die Untersuchung wird typischerweise stehend oder in Linksseitenlage durchgeführt. Als klassischer Befund zeigen sich Spiegel und stehende Dünndarmschlingen bei überwiegender Dünndarmbeteiligung sowie ein überblähtes Zökum bei Fokus im Kolon (Abb. 1).

- Cave. Eine Unterscheidung zwischen mechanischer Obstruktion und paralytischem lleus ist in der Abdomenübersicht nicht sicher möglich.

Hilfreich in der Differenzierung der mechanischen Obstruktion vom paralytischen Ileus kann die Sonografie des Abdomens sein. Bei abdominellen Notfällen ist die Sonografie oft die Methode der ersten Wahl. Bei Darmpassagestörungen kann der Ultraschall des Abdomens flüssigkeitsgefüllte Darmschlingen nachweisen. Freie Flüssigkeit im Abdomen kann ein Hinweis für eine stattgehabte Darmperforation sein. Fehlende Peristaltik deutet auf eine Paralyse des Darms hin.

Allerdings ist die Beurteilbarkeit insbesondere im späteren Stadium durch Darmgasüberlagerung eingeschränkt. Die Untersuchung ist darüber hinaus sehr

\section{Hintergrund}

Laboruntersuchungen

Laboruntersuchungen helfen bei der Diagnosefindung kaum, stellen jedoch einen wichtigen Bestandteil des Workups dar. Insbesondere lässt sich damit der Schweregrad der Erkrankung abschätzen. 


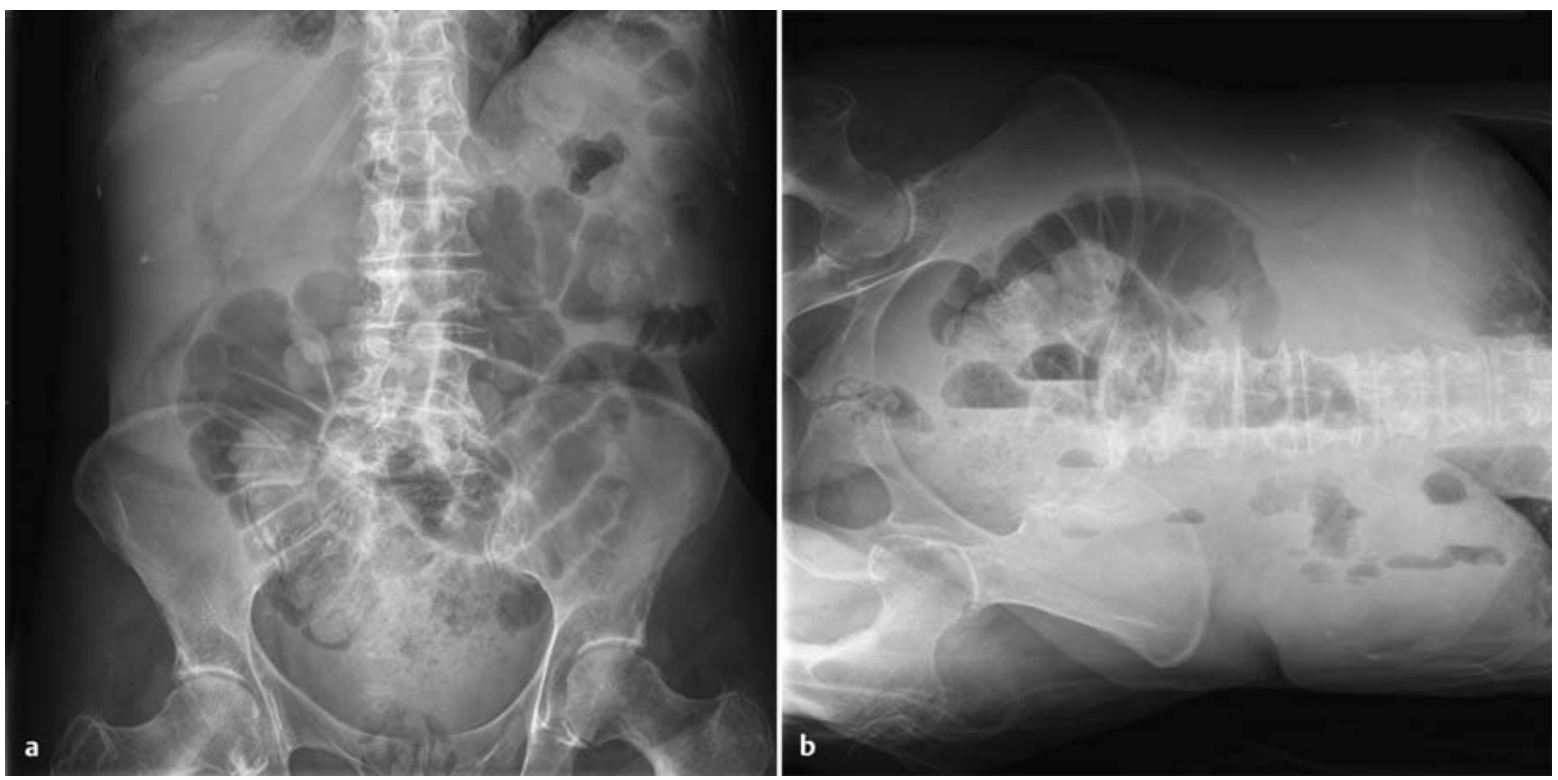

Abb. 1 - Ileus mit stehenden Schlingen und Flüssigkeitsspiegeln in der konventionellen Röntgen-Abdomen-Übersicht. a Röntgenübersicht des Abdomens im Stehen. b Röntgenübersicht des Abdomens in Linksseitenlage.
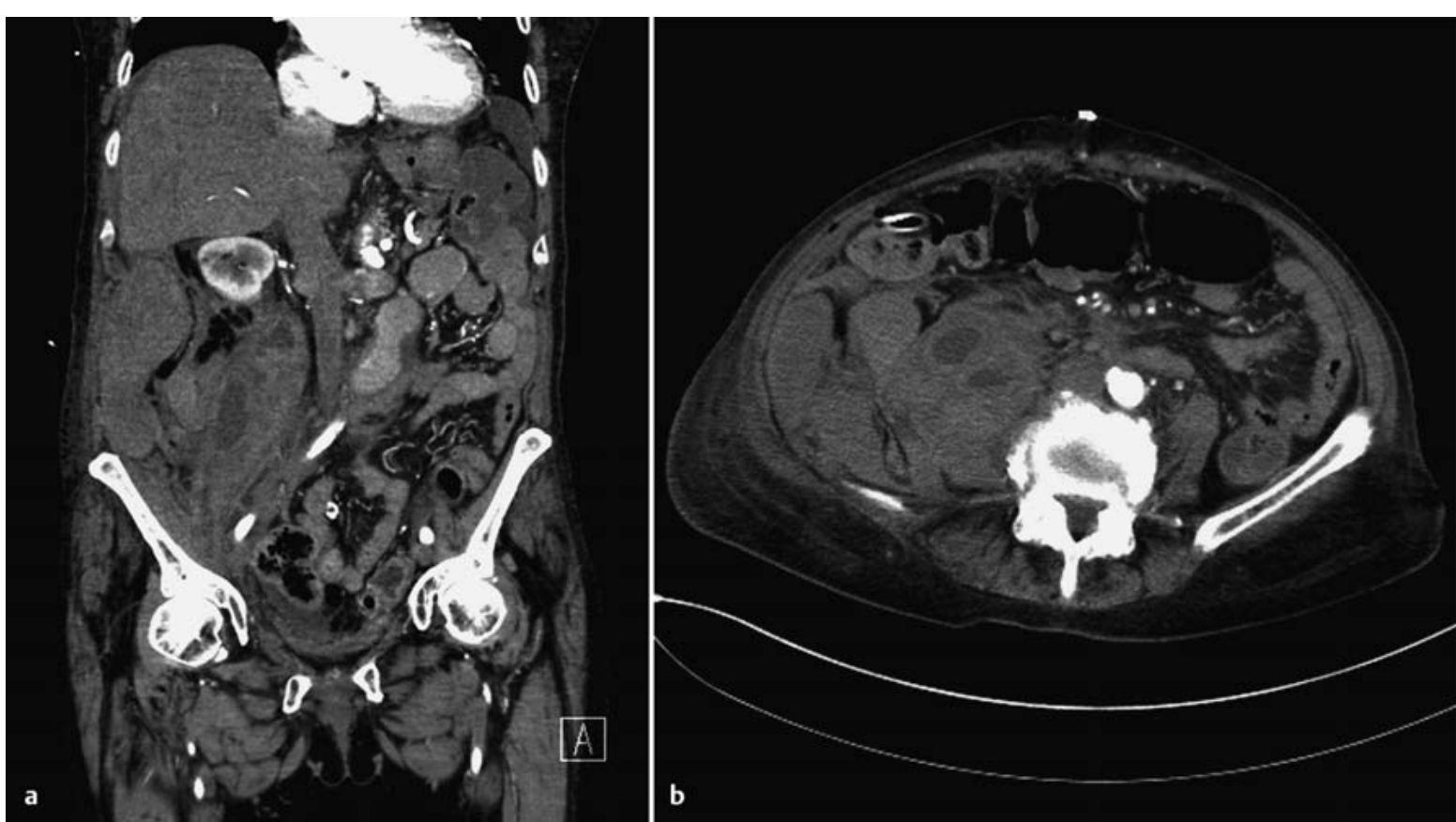

Abb. 2 - Computertomografie des Abdomens mit oralem und intravenösem Kontrastmittel derselben Patientin. Es zeigt sich ein ausgedehntes retroperitoneales Hämatom rechtsseitig als Ursache des paralytischen lleus. Auslösend war eine spontane Lumbalarterienblutung unter oraler Antikoagulation. a Koronale Schnittführung. b Axiale Schnittführung.

untersucherabhängig und schlecht demonstrierbar. Sie ist trotz flächendeckender Verfügbarkeit in dieser Situation nicht als Standard anzusehen.

Die Computertomografie (CT) des Abdomens kann neben der Diagnose der mechanischen Obstruktion und der des paralytischen Ileus auch in der Differenzialdiagnose und in der Abklärung möglicher Ursachen hilfreich sein. So spricht ein definierter Passagestopp (z.B. enge Ileumschlinge mit oral davon gelegener Distension) differenzialdiagnostisch für ein mechanisches Problem, ein ubiquitär dilatierter Dünndarm eher für einen paralytischen 
Ileus. Auslösende Faktoren für einen paralytischen Ileus können dargestellt werden, z. B. ein retroperitoneales Hämatom oder eine Pankreatitis (Abb. 2).

Zudem können mit der CT die mesenteriale Perfusion und die Durchblutung der Darmwand beurteilt werden. Die Untersuchung sollte deshalb wenn möglich immer mit oralem und intravenösem Kontrastmittel durchgeführt werden. Die rektale Kontrastmittelapplikation ist zur Beurteilung des Kolons ebenfalls hilfreich. Die in der Vergangenheit durchgeführten Kolonkontrasteinläufe mit wasserlöslichem Kontrastmittel sind aufgrund der nur sehr eingeschränkten Aussagekraft eigentlich obsolet, zumal das heute in der Breite verfügbare CT bei rektaler Füllung deutlich mehr Informationen liefert.

Die Magnetresonanztomografie spielt in der Akutdiagnostik der mechanischen Obstruktion sowie des paralytischen Ileus derzeit noch keine Rolle. Allerdings wird die mit endoluminal appliziertem Kontrastmittel kombinierte MR-Sellink-Untersuchung bei subakuten Stenosen des Dünndarms bei Morbus-Crohn-Patienten regelhaft eingesetzt.

Eine Koloskopie kann im Einzelfall für die Diagnosesicherung ebenfalls sinnvoll sein. Dieses diagnostische und gleichzeitig interventionelle Verfahren bietet den Vorteil, eine bestehende mechanische Obstruktion im distalen Kolon mittels Entlastungssonde oder Stent versorgen zu können. Beim paralytischen Ileus kann es notwendig sein, das Kolon durch Absaugung zu entlasten.

\section{Therapeutisches Vorgehen}

Therapieziele

Mechanische Obstruktion und paralytischer lleus

Die Therapie der mechanischen Obstruktion und des paralytischen lleus verfolgt zwei Ziele:

- die kausale Therapie mit Beseitigung der Ursache (sofern möglich)

- die supportive Therapie mit Behandlung der Folgen (lleuskrankheit)

\section{Therapeutisches Vorgehen beim paralytischen Ileus}

Der paralytische Ileus wird meist konservativ behandelt. Eine Indikation zur chirurgischen Intervention ergibt sich bei
- ausgeprägter Darmdistension mit Gefahr der Ischämie und Ruptur (Zökumdurchmesser $>10 \mathrm{~cm}$ ),

- operativ zu behandelnden auslösenden Faktoren (z.B. intraabdomineller Abszess, Anastomoseninsuffizienz) sowie

- toxischem Megakolon infolge einer Colitis ulcerosa.

Die konservative Therapie des paralytischen Ileus umfasst folgende wesentliche Punkte:

- Anlage einer Magensonde zur Entlastung des Magens und oberen Dünndarms sowie zur Vermeidung einer Aspiration

- Anlage eines Urinkatheters zur Bilanzierung. Über den Blasenkatheter kann zudem der intraabdominelle Druck gemessen und ein Kompartmentsyndrom rechtzeitig erkannt werden.

- Volumentherapie, Ausgleich der Elektrolytstörung und des Säure-Basen-Haushalts, intensivmedizinische Therapie eventueller Sepsisfolgen

- antibiotische Therapie bei bakterieller Translokation (z.B. Cephalosporin der dritten Generation, Metronidazol)

- ggf. das koloskopische Einbringen einer Entlastungssonde zur Dekompression des Kolons (zur Vermeidung von Drucknekrosen nicht länger als 72 Stunden belassen)

- prokinetische Therapie, laxierende Maßnahmen

- Beseitigung auslösender Faktoren, z.B. Absetzen einer auslösenden Medikation oder Therapie einer Urämie

- Aufnahme auf Intensivstation

Für die peristaltikanregende Therapie wird eine Vielzahl von Medikamenten verwendet, deren Nutzen in der Regel jedoch nicht hinreichend evidenzbasiert belegt ist. Die Applikation (Dosierung, Anwendungsdauer) ist zudem klinikindividuell sehr unterschiedlich. Es werden vor allem Cholinergika intravenös verwendet wie Neostigmin, welches die Kontraktilität von Dünn- und Dickdarm steigert (0,4-0,8 $\mathrm{mg}$ in $24 \mathrm{~h}$ oder 2,5 $\mathrm{mg}$ über 5-60 min). Unter der Therapie können Krämpfe und Erbrechen auftreten. Als weitere prokinetische Substanzen werden Dopaminantagonisten wie Metoclopramid oder Serotoninagonisten wie Cisaprid eingesetzt. Als Motilinagonist wird Erythromycin, ein Makrolidantibiotikum, verwendet (Off-Label-Use, 3-4-mal 200-250 mg i.v. oder 3-mal 0,5 g p.o.).

\section{Therapeutisches Vorgehen bei mechanischer Obstruktion}

Bei einer mechanischen Obstruktion hingegen ist die Therapie der Wahl meist die Operation oder die bereits oben beschriebenen interventionellen Verfahren. Letztere 


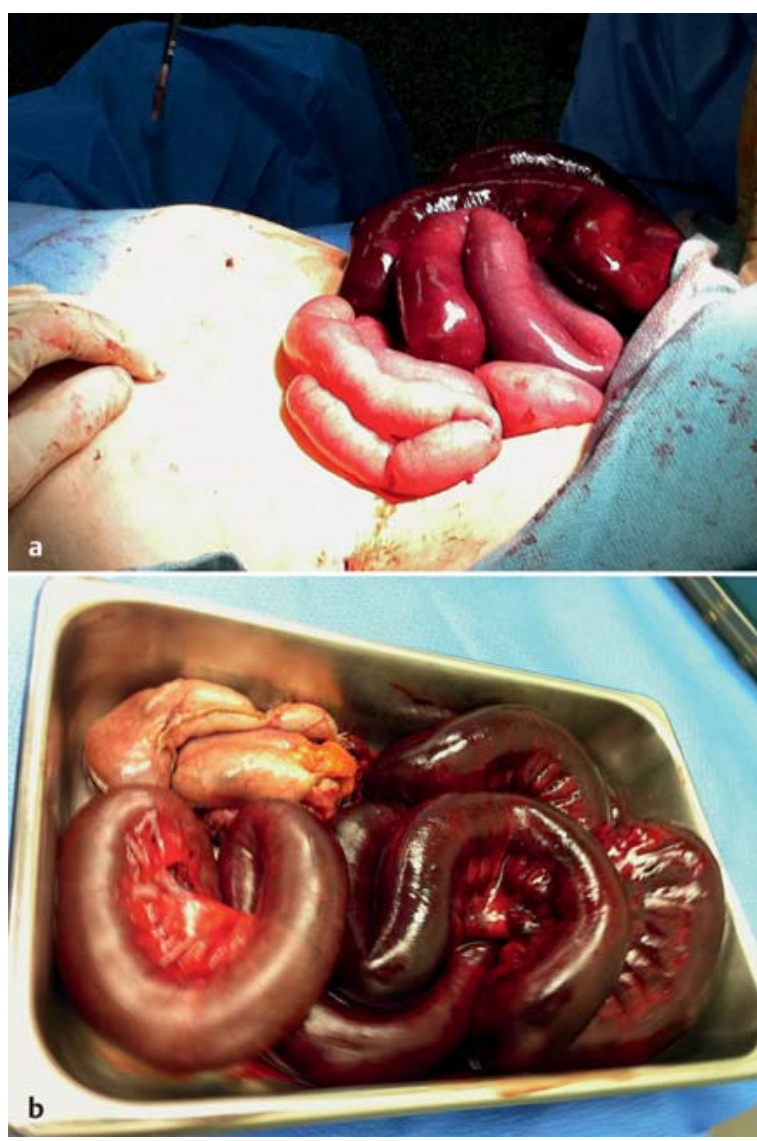

Abb. 3 - Mechanische Obstruktion. a Mechanische Obstruktion durch Strangulation des Dünndarms durch eine innere Hernie. b Resektat mit bereits nekrotischen Dünndarmanteilen.

dienen dabei häufig nur dem Bridging. Eine Operation kann in der Regel nicht vermieden werden. Sollte der klinische Befund soweit fortgeschritten sein, dass bereits eine Abwehrspannung vorliegt, ist die sofortige Operation indiziert. Korrespondieren die abgenommenen Laborparameter und die angefertigte Bildgebung mit dem klinischen Befund, so ist der Patient akut gefährdet und muss umgehend operiert werden. Dann muss befürchtet werden, dass bereits eine Peritonitis bzw. Strangulation mit Durchblutungsstörung vorliegt (Abb. 3).

Allgemeingültige Regeln, wann operiert werden soll, gibt es jedoch nicht. In Zusammenschau aller klinischen und apparativen Befunde muss für jeden einzelnen Patienten eine individuelle Entscheidung getroffen werden.

\section{Prognose}

Die Prognose der mechanischen Obstruktion und des paralytischen Ileus ist sowohl von der Grundkrankheit als auch von den systemischen Folgen der Krankheit abhän- gig. Generell wird bei Dünndarmbeteiligung eine Letalität von $5 \%$ angegeben, für den Dickdarm von 15 - 35\%, allerdings ohne Unterscheidung zwischen mechanischem und paralytischem Ileus. Problematisch sind die teilweise schwierige Differenzialdiagnose und das häufig schwer verlaufende und intensivpflichtige Krankheitsbild.

\section{Postoperativer lleus}

\section{Einleitung}

Der postoperative lleus (POI) ist eine Sonderform des paralytischen lleus, die nach operativen Eingriffen, vor allem in der Bauchhöhle, auftreten kann.

Er führt - selbst ohne das Auftreten der weiter oben beschriebenen schwerwiegenden Komplikationen - zu einer gesteigerten postoperativen Morbidität, hat meist einen verlängerten Krankenhausaufenthalt zur Folge und führt zu einem nicht unerheblichen Anstieg der Behandlungskosten.

Aus diesen finanziellen Kostenüberlegungen ergibt sich die Notwendigkeit, die postoperativen Liegezeiten weiter zu verkürzen und den postoperativen Ileus frühzeitig zu behandeln oder möglichst ganz zu vermeiden - ganz abgesehen von den schwerwiegenden Beeinträchtigungen des Wohlbefindens der betroffenen Patienten, die weiterhin im Zentrum ärztlichen Handelns stehen.

Die Remission des postoperativen lleus erfolgt bei leichtem Verlauf (unkomplizierter postoperativer Ileus) spontan innerhalb von zwei bis drei Tagen und stützt sich auf die Wiederherstellung und Normalisierung der physiologischen gastrointestinalen Peristaltik, die sich häufig durch das Auftreten von Darmgeräuschen und Flatulenz

\section{Hintergrund}

In den USA wurde für das Jahr 2000 angenommen, dass Motilitätstörungen nach Operationen im Bauchraum zu einem finanziellen Mehraufwand von einer Milliarde Dollar pro Jahr führen. Auch in Deutschland dürfte eine dreistellige Euro-Millionensumme pro Jahr überschritten werden, insbesondere, wenn auch indirekte Kosten wie beispielsweise die verlängerte Arbeitsunfähigkeit mit einbezogen werden. Dieser Aspekt der Behandlung gewinnt erheblich an Bedeutung, da nach Einführung der Diagnosis related Groups in Deutschland der wirtschaftliche Druck, zumindest kostenneutral zu arbeiten, zunimmt. 
ankündigt sowie durch die Toleranz oraler Nahrungszufuhr gekennzeichnet ist.

Die Erholung der gastrointestinalen Motilität folgt dabei einem relativ gut vorhersagbaren zeitlichen Muster: Die Wiederherstellung der Dünndarmfunktion erfolgt am schnellsten und durchschnittlich bereits fünf bis zehn Stunden nach dem chirurgischen Eingriff, wohingegen sich die Funktion des Magens erst nach 24-48 Stunden erholt und der Dickdarm sogar erst nach drei bis vier Tagen wieder vollständig funktionsfähig wird.

Die Wiederherstellung der Funktion des Dickdarms stellt somit den limitierenden Faktor innerhalb der Remission des postoperativen lleus dar.

Ergibt sich eine Erholung der Magen-Darm-Funktion nicht spontan, sondern zeigen sich im Gegenteil Symptome, die einen schweren paralytischen Verlauf des postoperativen Ileus vermuten lassen, müssen weitergehende therapeutische Konsequenzen ergriffen werden.

Neben der postoperativ standardmäßig durchgeführten Schmerztherapie kommt der Dekompression des MagenDarm-Trakts eine besondere Bedeutung zu. Bei fortgeschrittener Darmatonie bzw. bei länger bestehendem POI mit Übelkeit und Erbrechen erfolgt das Legen einer Magensonde, um eine Entlastung des Magens und Dünndarms zu erreichen und weitere Komplikationen wie eine drohende Aspiration zu vermeiden. Eine ausreichende Flüssigkeitszufuhr und der Ausgleich von Elektrolytimbalancen stehen nach wie vor an erster Stelle der Therapie. Darüber hinaus können Prokinetika und Abführmaßnahmen eingesetzt werden.

Generell haben alle beschriebenen Maßnahmen weitgehend einen eher supportiven Charakter. Diese Tatsache liegt darin begründet, dass nach wie vor ein nur unvollkommenes Verständnis des Krankheitsbilds existiert, sowohl hinsichtlich der einzelnen pathophysiologischen Mechanismen, die bei der Entstehung des postoperativen Ileus eine Rolle spielen, als auch hinsichtlich deren jeweiliger Gewichtung im zeitlichen Verlauf der Erkrankung.

\section{Grundlagen der Pathophysiologie des postoperativen Ileus}

In den letzten Jahren hat sich das Spektrum möglicher Ursachen für die Entstehung des postoperativen Ileus stark erweitert.

Neben einer Beteiligung sympathisch vermittelter Reflexe wurde auch die Wirkung inhibitorischer humoraler Sub- stanzen diskutiert, ebenso die Freisetzung von Noradrenalin aus der Darmwand, die Wirkung von zur Anästhesie eingesetzten Medikamenten und letztendlich auch die im Rahmen des postoperativen Ileus auftretenden Entzündungsvorgänge.

Seit der Einführung laparoskopischer Techniken erscheinen vermehrt Veröffentlichungen von Berichten, welche eine Verbindung zwischen minimalinvasiven Techniken mit einer verkürzten postoperativen Ileuserkrankung aufzeigen. Diese Ergebnisse legen nahe, dass der operative Eingriff per se wie auch die nachfolgende Schmerzmedikation eine wichtige Rolle bei der Verursachung des postoperativen Ileus spielen.

- Drei hauptsächliche Mechanismen werden im Rahmen der Entstehung des postoperativen lleus als ursächlich beteiligt angesehen und diskutiert: neurogene, inflammatorische und pharmakologische Mechanismen.

Im Rahmen der Frühphase des postoperativen Ileus scheint vor allem die Aktivierung spinaler wie auch supraspinaler adrenerger und nonadrenerger Signalwege eine Rolle zu spielen.

Ergänzend dazu konnte in erst kürzlich veröffentlichten Studien gezeigt werden, dass die ausgedehnte Spätphase des Ileus durch eine molekulare Entzündungsreaktion innerhalb der Darmwand verursacht wird, die in der Folge durch die Rekrutierung von Leukozyten in die Tunica muscularis der betroffenen Darmsegmente gekennzeichnet ist (Abb. 4). Diese Entzündungsreaktion beeinträchtigt die lokale neuromuskuläre Funktion und führt zur Aktivierung neurogener inhibitorischer Signalwege, durch die eine Hemmung der Motilität des gesamten Gastrointestinaltrakts vermittelt wird.

Die Anwendung von Opioiden zur postoperativen Schmerzkontrolle leistet ebenfalls einen nicht zu vernachlässigenden Beitrag, wenn man die Verringerung der propulsiven gastrointestinalen Motilität nach abdominalchirurgischen Eingriffen betrachtet.

- Die verschiedenen erwähnten Mechanismen können nicht unabhängig voneinander betrachtet werden. Vielmehr besteht eine enge Kooperation zwischen ihnen, wobei die Gewichtung eines jeden einzelnen Faktors im Verlauf der Erkrankung sicherlich variieren kann und eine beträchtliche Überlappung wie auch Interaktion zwischen den verschiedenen Mechanismen besteht. 


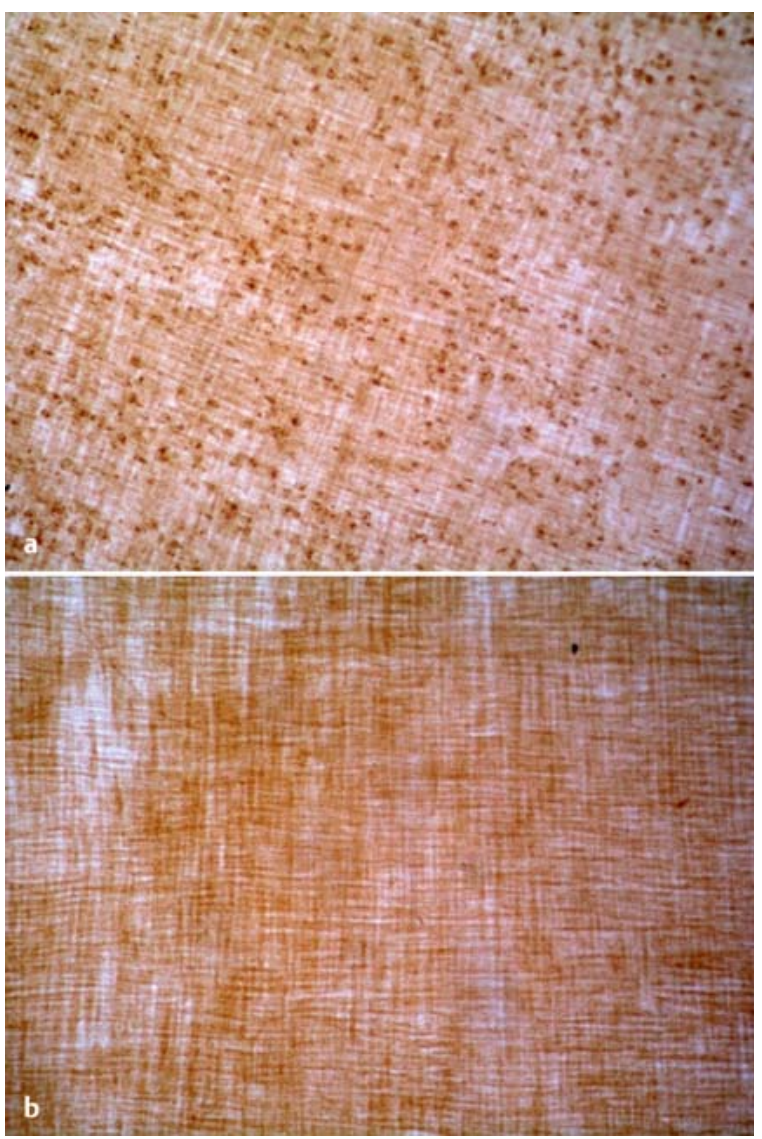

Abb. 4 - Die Abbildung zeigt einen lichtmikroskopischen Ausschnitt der Dünndarmmuskularis. Man sieht deutlich die massive Wanderung von Neutrophilen (Punkte) in die Darmwandmuskulatur als Zeichen einer ausgeprägten Entzündungsreaktion. a Beim postoperativem lleus neun Stunden nach einer Operation. b Kontrolle in 200-facher Vergrößerung.

\section{Die Wirkung von Anästhetika}

> Alle bekannten Substanzklassen von Anästhetika entfalten eine Wirkung auf die gastrointestinale Motilität. Dabei können sie ihren stärksten Einfluss auf Darmregionen nehmen, die in großem Maße von externer neuraler Steuerung abhängig sind wie der Dickdarm, der keine interzellulären Gap Junctions aufzuweisen hat und somit eine größere Anfälligkeit für die Wirkung inhibitorischer Anästhetika zeigt.

Eine Verzögerung der Magenentleerung kann ebenfalls nach Exposition gegenüber Anästhetika beobachtet werden, unabhängig davon, ob es sich nun um Atropin, Halothan oder Enfluran handelt. Die Konsequenzen einer verzögerten Magenentleerung sind mannigfaltig und reichen von einem erhöhten Aspirationsrisiko über postoperative Übelkeit und Erbrechen bis zur verzögerten Absorption anderer Medikamente.
Postoperative Epiduralanästhesie mit lokal wirkenden Anästhetika führt zu einer Blockade afferenter wie auch efferenter inhibitorischer Reflexe. Weiterhin kommt es zur Steigerung des Blutflusses im Splanchnikusgebiet oder auch zur Entwicklung antiinflammatorischer Effekte. Epidural applizierte Anästhetika besitzen zusätzlich den positiven Effekt, dass sie die afferenten Stimuli blockieren können, durch welche eine endokrine metabolische Stressantwort des Organismus als Reaktion auf den chirurgischen Eingriff getriggert wird - sie sind demnach in der Lage, die katabole Wirkung von Hormonen zu unterbinden, die normalerweise im Rahmen dieses Prozesses freigesetzt werden.

- Die Ergebnisse etlicher Studien zeigen: Im Vergleich zu einer alleinigen postoperativen systemischen Opioidtherapie führt die postoperative thorakal-epidurale Anwendung von Bupivacain-Hydrochlorid zu einer signifikanten Reduktion des Beschwerdebilds des postoperativen lleus.

Weiterhin konnte in drei von vier Studien nachgewiesen werden, dass nach epiduraler Applikation von Bupivacain die Erkrankungsdauer des postoperativen Ileus reduziert war - im Vergleich zur epiduralen Anwendung von Opioiden. Es existieren nur wenige prospektive Studien, die einen Vergleich der Effekte von alleiniger epiduraler Bupivacainapplikation mit der Kombination von epiduralem Bupivacain und Morphin anstellen. Die Ergebnisse dieser Studien zeigen, dass die alleinige Anwendung von epiduralem Bupivacain der genannten Kombinationstherapie mit Morphin überlegen ist, ohne dass nachteilige Effekte im Sinne einer geringeren postoperativen Schmerzreduktion zu erwarten sind.

- Generell scheint der Ort entscheidend zu sein, an dem die geplante Epiduralanästhesie zur Anwendung kommen soll: Im Gegensatz zu den Ergebnissen der bisher erwähnten Studien konnte bei einer lumbalen oder tiefen thorakalen epiduralen Anästhesie kein positiver Effekt für den Verlauf des postoperativen Ileus verzeichnet werden.

\section{Die Wirkung von endogenen und exogenen Opioiden}

Die pharmakologische Therapie des postoperativen Ileus gestaltet sich aufgrund des mangelnden Verständnisses seiner pathophysiologischen Ursachen eher schwierig.

Opioide werden nach abdominellen chirurgischen Eingriffen universell als Analgetika eingesetzt, führen unglücklicherweise aber häufig zu einer verlängerten Krankheitsdauer sowie zu einer Häufung von Komplikationen im Verlauf des postoperativen Ileus. 
- Seit Jahren gilt es als erwiesen, dass exogene Opioide eine Störung der normalen gastrointestinalen Motilität verursachen.

In-vivo-Studien zur Funktion des Dünndarms konnten zeigen, dass exogene $\delta$ - und $\mu$-Opioidrezeptor-Agonisten die peristaltische Aktivität zum Erliegen bringen und eine Steigerung der interdigestiven Kontraktilität bewirken. Gleichfalls wird häufig eine Verzögerung des intestinalen Transits wie auch eine Hemmung der Magenentleerung nach Applikation von Morphin beobachtet.

Endogene Opioide wie Enkephalin und Dynorphin konnten innerhalb des Plexus myentericus und der zirkulären Muskelschicht des Dünndarms nachgewiesen werden. Die Existenz dreier unterschiedlicher Typen von Opioidrezeptoren innerhalb des Gastrointestinaltrakts ist derzeit bekannt, wobei sich deren Klassifikation als $\boldsymbol{\delta}$-, $\boldsymbol{\mu}$ - und $\mathbf{\kappa - O p i o i d r e z e p t o r e n ~ a l l g e m e i n ~ d u r c h g e s e t z t ~}$ hat. Techniken, welche intrazelluläre In-vitro-Aufzeichnungen der Muskelaktivität verwenden, konnten die Wirkungsweise von Opioiden an glatten Muskelzellen offenlegen.

Durch die Ergebnisse dieser Studien kann man heute davon ausgehen, dass sowohl $\delta$ - als auch $\mu$-Opioidrezeptoren auf NO-freisetzenden NANC-Nerven (NANC: nonadrenerg, noncholinerg) vorhanden sind, welche die zirkuläre Muskelschicht innervieren. Bei Besetzung der $\delta$-Opioidrezeptoren durch beispielsweise Methionin-Enkephalin oder auch Dynorphin kommt es zu einer Abschwächung der Freisetzung inhibitorischer Neurotransmitter (wie NO) aus diesen Neuronen. Es wird daher angenommen, dass endogene wie exogene Opioide eine Minderung der NO-Freisetzung und anderer hemmender NANC-Transmitter bewirken, indem sie an Rezeptoren auf Nervenfasern innerhalb der zirkulären Muskelschicht ihre Wirkung entfalten. Dabei scheint diese Wirkung sowohl über $\delta$ - als auch über $\mu$-Opioidrezeptoren vermittelt zu sein.

\section{Therapeutisches Vorgehen beim POI}

Die Behandlung des POI wird in der Regel konservativ durchgeführt. Neben der Ableitung von Sekret über Magensonden kommen vor allem propulsive Medikamente zum Einsatz. Mögliche weitere therapeutische Ansätze sind

- Morphinreduktion,

- Epiduralkatheter,

- COX-2-Hemmer,

- Kaugummi,

- propulsive Therapie.
Risikofaktoren

Mögliche Wechselwirkungen der gängigen Medikamente

- Erythromycin: Motilinrezeptoragonist: propulsiv an Magen/Duodenum (Arrythmia, verlängerte QT-Zeit)

- Neostigmin: Cholinesterasehemmer: propulsiv (Bradykardie)

- Metoclopramid: Dopaminrezeptorantagonist: antiemetisch

- 5-HT4-Rezeptoragonist: propulsiv

- 5-HT-Rezeptorantagonist: antiemetisch (Parkinson)

- 5-HT4-Rezeptoragonist

Die peristaltikanregenden Medikamente weisen auch beim POI gewisse positive Effekte auf und werden von einer Vielzahl von Kliniken regelmäßig eingesetzt. Man sollte sich jedoch vor dem Einsatz der Medikamente nochmals deren Wechselwirkungs- und Nebenwirkungsprofil anschauen, um keine Patienten zu gefährden.

\section{Prophylaxe des POI}

Die Reduktion von Morphingaben sowie der Einsatz von Epiduralkathetern zur Blockierung neuronaler Reflexmechanismen können helfen, das Auftreten des POI abzuschwächen. Ganz entscheidend scheint jedoch die Minimierung des operativen Traumas zu sein. Durch schonende minimalinvasive Operationstechniken sollte das Auftreten des POI in Zukunft drastisch reduziert werden können. Katabole Ernährungszustände und Elektrolytimbalancen tragen ebenfalls stark zur Ausbildung des POI bei und sollten, wenn irgend möglich, vermieden werden.

\section{Zusammenfassung}

Häufigste Ursachen. Mechanische Obstruktion: Briden, Adhäsionen, Tumoren, Fremdkörper. Paralytischer Ileus: postoperativ, retroperitoneales Hämatom, Frakturen, Ogilvie-Syndrom, intra- oder retroperitoneale Infektionen, entzündliche Darmerkrankungen, medikamentös (Opioide, Katecholamine, Antidepressiva, Neuroleptika), metabolisch, toxisch, vaskulär (Mesenterialinfarkt).

Leitsymptome. Mechanische Obstruktion: Kolikartige abdominelle Schmerzen, Hyperperistaltik, Übelkeit und Erbrechen sowie Stuhlverhalt. Paralytischer Ileus: Singultus, schwallartiges Erbrechen, Stuhlverhalt, distendiertes Abdomen, auskultatorische Totenstille, diffuser Bauchschmerz, Hypovolämie mit Tachykardie und Hypotonie, Schock. 
Diagnostisches Vorgehen. Abdomenübersicht, Sonografie, ggf. Computertomografie des Abdomens, Labor, Blutgasanayse, koloskopische Absaugung, ggf. Stent

Therapeutisches Vorgehen. 1. Kausale Therapie der Ursache. 2. Behandlung der Ileuskrankheit: häufig intensivmedizinische Therapie, Anlage einer Magensonde, Urinkatheter, Volumen-/Elektrolyttherapie, Antibiotika, ggf. Kolonentlastungssonde, prokinetische Therapie, Laxanzien, Beseitigung auslösender Faktoren (z.B. Medikation, Stoffwechsel).

Sonderform postoperativer lleus. Neuronale und inflammatorische Prozesse tragen zur Ausbildung des POI bei. Die Therapie erfolgt analog zum paralytischen Ileus. Die Prophylaxe durch Reduzierung des chirurgischen Traumas ist vielversprechend.

\section{Zum Weiterlesen und Vertiefen}

Bauer AJ, Boeckxstaens GE. Mechanisms of postoperative ileus. Neurogastroenterol Motil 2004; 16 (Suppl 2): 54-60

Burchardi H, Larsen R, Marx G, Muhl E, Schölmerich J, Hrsg. Die Intensivmedizin. 11. Aufl. Berlin, Heidelberg: Springer; 2011

Jauch KW, Mutschler W, Hoffmann JN, Kanz KG, Hrsg. Chirurgie Basisweiterbildung. 2. Aufl. Berlin, Heidelberg: Springer; 2013

Kreis ME, Jauch KW. lleus aus chirurgischer Sicht. Differenzialdiagnose und therapeutische Konsequenzen. Chirurg 2006; 77: 883 -888

Landwer P. Radiologische Akutdiagnostik bei Darmobstruktion. Chirurg 2006; 77: 889-897

Livingston EH, Passaro EP jr. Postoperative ileus. Dig Dis Sci 1990; 35: $121-132$

Luckey A, Livingston E, Tache Y. Mechanisms and treatment of postoperative ileus. Arch Surg 2003; 138: 206-214

Plusczyk T, Bolli M, Schilling M. Ileuskrankheit. Chirurg 2006; 77: 898 903

Riede UN, Schäfer HE, Werner M. Allgemeine und spezielle Pathologie. 5. Aufl. Stuttgart: Thieme; 2004

Siewert JR, Rothmund M, Schumpelick V, Hrsg. Praxis der Viszeralchirurgie. Gastroenterologische Chirurgie. 3. Aufl. Berlin, Heidelberg: Springer; 2011
Interessenkonflikt: Die Autoren bestätigen, dass kein Interessenkonflikt vorliegt.

\section{Korrespondenzadresse}

Priv.-Doz. Dr. med. Mario Müller

Klinik für Allgemein-, Thorax- und Gefäßchirurgie

Charité Berlin - Campus Benjamin Franklin

Hindenburgdamm 30

12200 Berlin

Telefon: 030/450522722

Fax: $\quad 030 / 450522902$

E-Mail: mueller.mario@charite.de 


\section{CME-Fragen}

\section{CME•thieme.de}

\section{CME-Teilnahme}

- Viel Erfolg bei Ihrer CME-Teilnahme unter http://cme.thieme.de

- Bitte informieren Sie sich vorab online über die Gültigkeitsdauer.

- Sollten Sie Fragen zur Online-Teilnahme haben, unter http://cme.thieme.de/hilfe finden Sie eine ausführliche Anleitung.

Welches diagnostische Mittel sollte gewählt werden, um die Differenzialdiagnosen der mechanischen Obstruktion zu untersuchen?
A Ultraschall
B CT
C Magnetresonanztomografie
D Koloskopie
E Laparoskopie

Welches sind keine klinischen Symptome des paralytischen Ileus?
A lebhafte Darmgeräusche
B abdominelle Distension
C Singultus
D schwallartiges Erbrechen
E Stuhlverhalt

\section{Welches sind keine} typischen Folgeprobleme der lleuskrankheit?
A Hypovolämie
B Niereninsuffizienz
C Diarrhoe
D Aspirationspneumonie bei Erbrechen
E hämodynamische Instabilität

Wie hoch ist die Letalität bei Dünndarmobstruktion?
A $5 \%$
B $1 \%$
C $50 \%$
D $80 \%$
E $25 \%$

Welches ist keine mögliche Ursache der mechanischen Obstruktion?
A Briden
B Adhäsionen
C Tumoren
D Fremdkörper
E viraler Infekt 
Welchen Nutzen bietet die Koloskopie in der Behandlung des mechanischen lleus nicht?
A koloskopische Dekompression
B Stentapplikation
C Entlastungssondenanlage
D Ausschluss Divertikulose
E Ausschluss eines Tumorleidens

Welche Laborparameter können in der diagnostischen Abklärung der lleuskrankheit nicht hilfreich sein?
A Kreatinin
B International normalized Ratio
C Laktat
D thyroideastimulierendes Hormon
E Kalium

Welche Fragestellungen können mit der Ultraschalluntersuchung in der Situation des mechanischen Ileus nicht beantwortet werden?
A fehlende Peristaltik
B freie Luft
C freie Flüssigkeit
D Hyperperistaltik
E Raumforderung

Welches der genannten Maßnahmen wird nicht zur Therapie des paralytischen lleus eingesetzt?
A Anlage einer Magensonde
B Volumenrestriktion
C antibiotische Therapie
D prokinetische Maßnahmen
E koloskopische Absaugung

Bei einer hohen mechanischen Obstruktion kann folgendes Symptom fehlen?
A Stuhlverhalt
B Übelkeit
C Erbrechen
D abdominelle Schmerzen
E Singultus 